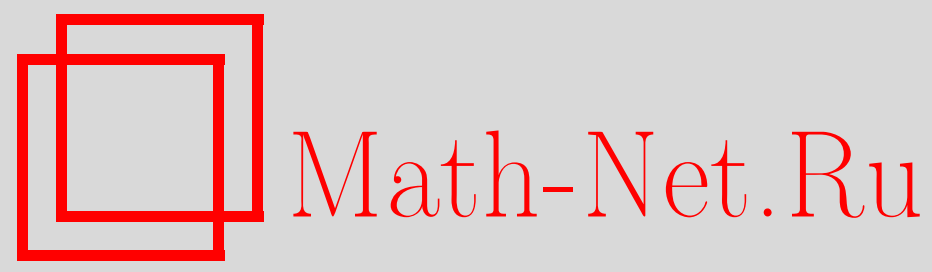

А. К. Свинин, Инвариантные совместные решения эволюционных уравнений интегрируемых иерархий, Матем. заметки, 2003, том 74, выпуск 1, 99-107

DOI: https://doi.org/10.4213/mzm250

Использование Общероссийского математического портала Math-Net.Ru подразумевает, что вы прочитали и согласны с пользовательским соглашением http://www . mathnet.ru/rus/agreement

Параметры загрузки:

IP : 54.198 .187 .58

26 апреля 2023 г., 05:56:47

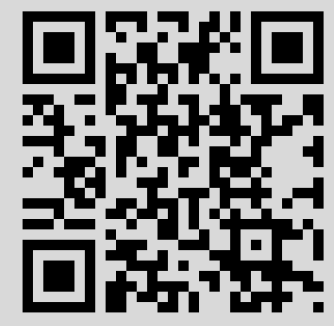




\title{
ИНВАРИАНТНЫЕ СОВМЕСТНЫЕ РЕШЕНИЯ ЭВОЛЮЦИОННЫХ УРАВНЕНИЙ ИНТЕГРИРУЕМЫХ ИЕРАРХИЙ
}

\author{
А. К. Свинин
}

Построены группы классических точечных симметрий для совместных пар эволюционных уравнений (систем уравнений) интегрируемых иерархий, связанных со вспомогательным уравнением метода обратной задачи второго порядка. Для двух случаев: иерархии уравнений Кортевега-де Фриза $(\mathrm{K} \Phi)$ и систем уравнений Каупа, строятся совместные решения, инвариантные относительно групп симметрий. Задача построения этих решений сводится, соответственно, к первому и второму уравнению Пенлеве с зависимостью от некоторого параметра. При этом, уравнения Пенлеве дополняются линейными эволюционными уравнениями, определяющими деформацию решения соответствующего уравнения Пенлеве.

Библиограбия: 5 названий.

1. Введение. Групповой анализ дифференциальных уравнений математической физики, как известно, играет существенную роль при описании общего множества решений, при выделении частных инвариантных решений, при установлении взаимосвязи между симметриями и законами сохранения и т.д. [1], [2].

Интегрируемые эволюционные уравнения, такие как уравнение КдФ, с групповой точки зрения имеют отличительную особенность: они включаются в иерархию коммутируюших совместньх уравнений (систем уравнений)

$$
\mathbf{u}_{t_{m}}=\mathbf{K}_{m}[\mathbf{u}], \quad\left[\mathbf{K}_{n}, \mathbf{K}_{m}\right]=0
$$

и поэтому имеет смысл рассматривать совместные решения $\mathbf{u}\left(x, t_{2}, t_{3}, \ldots\right)$.

В данной статье рассматриваются интегрируемые иерархии эволюционных уравнений, связанные со вспомогательным уравнением второго порядка (1). В п. 2 строятся однопараметрические групы линейных точечных преобразований симметрии для совместной пары эволюционных уравнений в каждой иерархии. Построение основано на трансформационньх свойствах рекурсионного оператора, которьй для этих иерархий известен в явной форме. В п. 3 и 4 строятся совместные решения уравнений иерархии КдФ и Каупа, инвариантные относительно данных групп преобразований. Подстановки, полученные с помощью стандартных методов [1], [2], в результате сводят задачу построения этих решений к первому и второму уравнению Пенлеве с зависимостью от некоторого параметра и дополнительному линейному эволюционному уравнению.

2. Интегрируемые иерархии и однопараметрические группы точечных преобразований. Известно, что со вспомогательным линейным уравнением метода обратной задачи

$$
\psi_{x x}(x, \lambda)+\left(u(x, \lambda)+(-\lambda)^{n}\right) \psi(x, \lambda)=0
$$


где $u(x, \lambda)=\sum_{i=1}^{n} u_{i}(x)(-\lambda)^{i-1}$, ассоциируется интегрируемая иерархия совместных эволюционных уравнений (систем уравнений) [3]

$$
\mathbf{u}_{t_{m}}=\mathbf{K}_{m}[\mathbf{u}]=\Lambda^{m-1}[\mathbf{u}] \mathbf{u}_{x}, \quad \mathbf{u}=\left(u_{1}, \ldots, u_{n}\right)^{T}
$$

с рекурсионным оператором

$$
\Lambda[\mathbf{u}]=\left(\begin{array}{ccccc}
0 & 0 & \ldots & 0 & \frac{1}{4} \partial_{x}^{2}+u_{1}+\frac{1}{2} u_{1 x} \partial_{x}^{-1} \\
-1 & 0 & \ldots & 0 & u_{2}+\frac{1}{2} u_{2 x} \partial_{x}^{-1} \\
0 & -1 & \ddots & \vdots & u_{3}+\frac{1}{2} u_{3 x} \partial_{x}^{-1} \\
\vdots & \ddots & \ddots & 0 & \vdots \\
0 & \ldots & 0 & -1 & u_{n}+\frac{1}{2} u_{n x} \partial_{x}^{-1}
\end{array}\right)
$$

Действие оператора интегрирования $\partial_{x}^{-1}$ определим следуюшим образом. Пусть $A_{\mathbf{u}}^{0} \subset A_{\mathbf{u}}$ - кольцо дифференциальных многочленов от $u_{i}$ с нулевыми свободными членами. Потребуем, чтобы $\partial_{x}^{-1}(f) \in A_{\mathbf{u}}^{0}$ для любого $f=f[\mathbf{u}] \in \operatorname{Im} \partial_{x} \subset A_{\mathbf{u}}^{0}$. В частности, если функции $u_{i}=u_{i}(x)$ принадлежат пространству Шварца $\mathscr{S}(\mathbb{R})$, то

$$
\partial^{-1}(\cdot) \stackrel{\text { def }}{=} \int_{-\infty}^{x} \cdot d x^{\prime} .
$$

После определения оператора интегрирования $\partial_{x}^{-1}$ рекурсионньй оператор однозначно определяет бесконечный набор коммутирующих векторных полей $\mathbf{K}_{m}[\mathbf{u}]=\Lambda^{m-1} \mathbf{u}_{x}$, $m \in \mathbb{N}$.

Очевидно, что сдвиг спектрального параметра в уравнении (1) $\bar{\lambda}=\lambda-\varepsilon$ оставляет инвариантным вид этого уравнения. Исходя из этого факта, определим однопараметрическое линейное преобразование зависимых переменных с помошью соотношения

$$
\sum_{i=1}^{n} u_{i}(x)(-\lambda)^{i-1}+(-\lambda)^{n}=\sum_{i=1}^{n} \bar{u}_{i}(x)(-\lambda+\varepsilon)^{i-1}+(-\lambda+\varepsilon)^{n} .
$$

Приравнивая коэффициенты при одинаковых степенях $\lambda$, получим

$$
\begin{gathered}
u_{1}=F_{1}(\overline{\mathbf{u}}, \varepsilon)=\sum_{i=1}^{n} \bar{u}_{i} \varepsilon^{i-1}+\varepsilon^{n}, \\
u_{i+1}=F_{i+1}(\overline{\mathbf{u}}, \varepsilon)=\frac{1}{i !} \frac{\partial^{i} F_{1}(\overline{\mathbf{u}}, \varepsilon)}{\partial \varepsilon^{i}}, \quad i=1, \ldots, n-1 .
\end{gathered}
$$

Из определения этого преобразования очевидно, что

$$
\bar{u}_{1}=F_{1}(\mathbf{u},-\varepsilon), \quad \bar{u}_{i+1}=F_{i+1}(\mathbf{u},-\varepsilon), \quad i=1, \ldots, n-1 .
$$

Также очевидно, что преобразование (3) можно переписать в векторном виде

$$
\mathbf{u}=\mathbf{F}(\overline{\mathbf{u}}, \varepsilon)=A(\varepsilon) \overline{\mathbf{u}}+d(\varepsilon),
$$

где $A(\varepsilon)$ - верхнетреугольная матрища с единицами на диагонали и $d(\varepsilon)$ - вектор. Матричные элементы $A(\varepsilon)$ и компоненты вектора $d(\varepsilon)$, как нетрудно убедиться, определяются следующими формулами:

$$
A_{i k}(\varepsilon)=\left(\begin{array}{c}
i-1 \\
k-1
\end{array}\right) \varepsilon^{k-i}, \quad k>i, \quad d_{i}(\varepsilon)=\left(\begin{array}{c}
i-1 \\
n
\end{array}\right) \varepsilon^{n-i+1} .
$$

Тогда

$$
\overline{\mathbf{u}}=\mathbf{F}(\mathbf{u},-\varepsilon)=A(-\varepsilon) \mathbf{u}+d(-\varepsilon) .
$$


Лемма. Выполняется соотношение

$$
\left.\Lambda[\mathbf{u}]\right|_{\mathbf{u}=F(\overline{\mathbf{u}}, \varepsilon)}=A(\varepsilon)(\Lambda[\overline{\mathbf{u}}]+\varepsilon) A^{-1}(\varepsilon) .
$$

Доказательство этой леммы приведено в конце статьи.

Формула (5) определяет закон преобразования рекурсионного оператора. Однако следует заметить, что оператор интегрирования $\partial_{x}^{-1}$, фигурирующий в $\Lambda[\overline{\mathbf{u}}]$, действует в кольце дифференциальных многочленов от преобразованных переменных $\bar{u}_{i}$ и его действие (выбор постоянной интегрирования) должно быть согласовано с преобразованием (4). Пусть $\mathbf{F}_{*}: A_{\mathbf{u}} \rightarrow A_{\overline{\mathbf{u}}}$ и $\mathbf{F}_{*}^{-1}: A_{\overline{\mathbf{u}}} \rightarrow A_{\mathbf{u}}$ - отображения колец дифференциальных многочленов, порожденные преобразованием (4). Тогда постоянная интегрирования однозначно определяется условием

$$
\mathbf{F}_{*}^{-1} \circ \partial_{x}^{-1}(f) \in A_{\mathbf{u}}^{0},
$$

для любого $f[\overline{\mathbf{u}}] \in \operatorname{Im} \partial_{x} \subset A_{\overline{\mathbf{u}}}^{0}$.

Таким образом, вычисляя векторные поля $\Lambda^{r}[\overline{\mathbf{u}}] \overline{\mathbf{u}}_{x}$, необходимо учитывать условие (6). Например,

$$
\Lambda[\overline{\mathbf{u}}] \overline{\mathbf{u}}_{x}=K_{2}[\overline{\mathbf{u}}]+\frac{n}{2} \varepsilon \overline{\mathbf{u}}_{x},
$$

так как учитьвается, что $\partial_{x}^{-1}\left(\bar{u}_{n x}\right)=\bar{u}_{n}+n \varepsilon$, потому что $\mathbf{F}_{*}^{-1}\left(\bar{u}_{n}+n \varepsilon\right)=u_{n} \in A_{\mathbf{u}}^{0}$. Для вычисления $\Lambda^{2}[\overline{\mathbf{u}}] \overline{\mathbf{u}}_{x}$ требуются две вспомогательные формулы:

$$
\partial_{x}^{-1}\left(\frac{1}{4} \bar{u}_{x x x}+\frac{3}{2} \bar{u} \bar{u}_{x}\right)=\frac{1}{4} \bar{u}_{x x}+\frac{3}{4} \bar{u}^{2}-\frac{3}{4} \varepsilon^{2}
$$

для $n=1$ и

$$
\partial_{x}^{-1}\left(-\bar{u}_{n-1, x}+\frac{3}{2} \bar{u}_{n} \bar{u}_{n x}\right)=-\bar{u}_{n-1}+\frac{3}{4} \bar{u}_{n}^{2}+\left(\frac{1}{2} n(n-1)-\frac{3}{4} n^{2}\right) \varepsilon^{2}
$$

для $n \geqslant 2$, которые следуют из условия (6). Используя формулы (7), (8) и (9), получаем

$$
\begin{aligned}
\Lambda^{2}[\overline{\mathbf{u}}] \overline{\mathbf{u}}_{x} & =\Lambda[\overline{\mathbf{u}}] K_{2}[\overline{\mathbf{u}}]+\frac{n}{2} \varepsilon \Lambda[\overline{\mathbf{u}}] \overline{\mathbf{u}}_{x} \\
& =\mathbf{K}_{3}[\overline{\mathbf{u}}]+\left(\frac{1}{2} n(n-1)-\frac{3}{4} n^{2}\right) \varepsilon^{2} \overline{\mathbf{u}}_{x}+\frac{n}{2} \varepsilon\left(\mathbf{K}_{2}[\overline{\mathbf{u}}]+\frac{n}{2} \varepsilon \overline{\mathbf{u}}_{x}\right) \\
& =\mathbf{K}_{3}[\overline{\mathbf{u}}]+\frac{n}{2} \varepsilon \mathbf{K}_{2}[\overline{\mathbf{u}}]+\left(\frac{1}{2} n(n-1)-\frac{1}{8} n^{2}\right) \varepsilon^{2} \overline{\mathbf{u}}_{x} .
\end{aligned}
$$

Используя лемму и формулы $(7),(10)$, получаем эволюционные уравнения на преобразованные функции $\bar{u}_{i}\left(x, t_{2}, t_{3}\right)$

$$
\begin{aligned}
\overline{\mathbf{u}}_{t_{2}}= & (\Lambda[\overline{\mathbf{u}}]+\varepsilon) \overline{\mathbf{u}}_{x}=\mathbf{K}_{2}[\overline{\mathbf{u}}]+\left(\frac{n}{2}+1\right) \varepsilon \overline{\mathbf{u}}_{x}, \\
\overline{\mathbf{u}}_{t_{3}}= & (\Lambda[\overline{\mathbf{u}}]+\varepsilon)^{2} \overline{\mathbf{u}}_{x}=\Lambda^{2}[\overline{\mathbf{u}}] \overline{\mathbf{u}}_{x}+2 \varepsilon \Lambda[\overline{\mathbf{u}}] \overline{\mathbf{u}}_{x}+\varepsilon^{2} \overline{\mathbf{u}}_{x} \\
= & \mathbf{K}_{3}[\overline{\mathbf{u}}]+\frac{n}{2} \varepsilon \mathbf{K}_{2}[\overline{\mathbf{u}}]+\left(\frac{1}{2} n(n-1)-\frac{1}{8} n^{2}\right) \varepsilon^{2} \overline{\mathbf{u}}_{x} \\
& +2 \varepsilon\left(\mathbf{K}_{2}[\overline{\mathbf{u}}]+\frac{n}{2} \varepsilon \overline{\mathbf{u}}_{x}\right)+\varepsilon^{2} \overline{\mathbf{u}}_{x} \\
& \quad \mathbf{K}_{3}[\overline{\mathbf{u}}]+\left(\frac{n}{2}+2\right) \mathbf{K}_{2}[\overline{\mathbf{u}}]+\frac{1}{2}\left(\frac{n}{2}+1\right)\left(\frac{n}{2}+2\right) \overline{\mathbf{u}}_{x} .
\end{aligned}
$$


Преобразование зависимых переменных (4) возможно дополнить преобразованием независимых переменньх $\left(x, t_{2}, t_{3}\right)$ так, чтобы в результате получить преобразование симметрии для пары совместных уравнений (систем уравнений) в иерархии. А именно, пусть

$$
\left(\begin{array}{c}
\bar{x} \\
\bar{t}_{2} \\
\bar{t}_{3}
\end{array}\right)=B(\varepsilon)\left(\begin{array}{c}
x \\
t_{2} \\
t_{3}
\end{array}\right)=\left(\begin{array}{ccc}
1 & \left(\frac{n}{2}+1\right) \varepsilon & \frac{1}{2}\left(\frac{n}{2}+1\right)\left(\frac{n}{2}+1\right) \varepsilon^{2} \\
0 & 1 & \left(\frac{n}{2}+1\right) \varepsilon \\
0 & 0 & 1
\end{array}\right)\left(\begin{array}{c}
x \\
t_{2} \\
t_{3}
\end{array}\right) .
$$

Матрица $B(\varepsilon)$, как нетрудно убедиться, удовлетворяет групповому соотношению

$$
B(\varepsilon) B\left(\varepsilon_{1}\right)=B\left(\varepsilon+\varepsilon_{1}\right) .
$$

Прямыми вычислениями можно убедиться в том, что

$$
\overline{\mathbf{u}}_{\bar{t}_{2}}=\mathbf{K}_{2}[\overline{\mathbf{u}}], \quad \overline{\mathbf{u}}_{\bar{t}_{3}}=\mathbf{K}_{3}[\overline{\mathbf{u}}] .
$$

Таким образом, можно сформулировать

УТВЕРЖДЕНИЕ 1. Линейные точечные преобразования переменных по формулам (4), (11) образуют однопараметрическую группу симметрий для совместной пары уравнений - первых двух нетривиальных членов в иерархии уравнений (2).

3. Инвариантные решения уравнений иерархии Кд $\Phi$. Рассмотрим пару уравнений в интегрируемой иерархии КдФ

$$
\begin{aligned}
& u_{t_{2}}=K_{2}[u]=\frac{1}{4} u_{x x x}+\frac{3}{2} u u_{x} \\
& u_{t_{3}}=K_{3}[u]=\frac{1}{16} u_{x}^{(V)}+\frac{5}{8} u u_{x x x}+\frac{5}{4} u_{x} u_{x x}+\frac{15}{8} u^{2} u_{x} .
\end{aligned}
$$

В этом случае формулы преобразования (4), (11) имеют следующий вид:

$$
\bar{u}=u-\varepsilon, \quad \bar{x}=x+\frac{3}{2} \varepsilon t_{2}+\frac{15}{8} \varepsilon^{2} t_{3}, \quad \bar{t}_{2}=t_{2}+\frac{5}{2} \varepsilon t_{3}, \quad \bar{t}_{3}=t_{3} .
$$

Дифференциальными инвариантами относительно образующей этой групшы

$$
\mathbf{v}=-\frac{\partial}{\partial u}+\frac{5}{2} t_{3} \frac{\partial}{\partial t_{2}}+\frac{3}{2} t_{2} \frac{\partial}{\partial x}
$$

как нетрудно проверить, являются

$$
\xi=t_{3}, \quad z=x t_{3}-\frac{3}{10} t_{2}^{2}, \quad w=t_{3} u^{2}+\frac{4}{5} t_{2} u+\frac{8}{15} x .
$$

Построим совместное решение уравнений $(12),(13)$, инвариантное относительногруппы преобразований (14). Подстановка для $u$ определяется из квадратного уравнения

$$
t_{3} u^{2}+\frac{4}{5} t_{2} u+\left(\frac{8}{15} x-w\right)=0
$$

Решение (15) имеет вид

$$
u=-\frac{2 t_{2}}{5 t_{3}} \pm \frac{\left(t_{3} w-\frac{8}{15} z\right)^{1 / 2}}{t_{3}}, \quad w=w(z, \xi)
$$


Сделаем подстановку $v=v(z, \xi)=\left(\xi w-\frac{8}{15} z\right)^{1 / 2}$. Очевидно, что выбор знака в (16) не играет роли. Поэтому, без ограничения обшности, выберем знак “+”. Подставляя (16) в (12), получаем

$$
v_{z z}=-\frac{1}{\xi^{3}}\left(3 v^{2}+\frac{8}{5} z+c(\xi)\right)
$$

где $c(\xi)$ - произвольная функция, получающаяся в результате однократного интегрирования по $z$. Подстановка (16) в уравнение КдФ пятого порядка (13), как нетрудно проверить, с учетом (17) дает уравнение эволюционного типа

$$
v_{\xi}=-\left(\frac{6 z}{5 \xi}+\frac{c(\xi)}{8 \xi}\right) v_{z}+\frac{3}{5 \xi} v .
$$

Прямыми вычислениями нетрудно убедиться, что условие совместности $v_{z z \xi}=v_{\xi z z}$ выполняется при условии, что вьполняется уравнение $\xi c^{\prime}(\xi)=c(\xi)$. Решение этого уравнения - линейная однородная функция по $\xi$. Пусть $c=8 \mu \xi$ с произвольной постоянной $\mu$. Таким образом, доказано

УТВЕРЖДЕНИЕ 2. Подстановка

$$
u\left(x, t_{2}, t_{3}\right)=\frac{-2 t_{2}+5 v(z, \xi)}{5 \xi}, \quad \xi=t_{3}, \quad z=x t_{3}-\frac{3}{10} t_{2}^{2}
$$

дает совместное решение (12), (13), инвариантное относительно группы преобразований (14) при условии, что $v=v(z, \xi)$ удовлетворяет совместной системе уравнений

$$
\begin{gathered}
v_{z z}=-\frac{1}{\xi^{3}}\left(3 v^{2}+\frac{8}{5} z+8 \mu \xi\right) \\
v_{\xi}=-\left(\frac{6 z}{5 \xi}+\mu\right) v_{z}+\frac{3}{5 \xi} v
\end{gathered}
$$

где $\mu$ - постоянная.

При каждом значении $\xi_{0} \neq 0$ уравнение $(18)$ является первым уравнением Пенлеве на функцию $v(z)=v\left(z, \xi_{0}\right)$. Коэффициенты $a_{k}$ "общего" решения (18) с полюсной особенностью в точке $z_{0}$

$$
v(z)=\frac{1}{\left(z-z_{0}\right)^{2}} \sum_{k=0}^{\infty} a_{k}\left(z-z_{0}\right)^{k}
$$

определяются, как известно, из рекурсионного соотношения вида

$$
(k+1)(k-6) a_{k}=f_{k}\left(a_{0}, \ldots, a_{k-1}\right), \quad k \geqslant 1,
$$

с резонансами при $k=-1$ и $k=6$. При этом $a_{1}=a_{2}=a_{3}=0$. В случае системы уравнений $(18),(19)$ очевидно, что $z_{0}$ и коэффициенты $a_{k}$ зависят от эволюционного параметра $\xi$. Несложные вычисления дают

$$
a_{0}=-2 \xi^{3}, \quad a_{4}=\frac{4}{5 \xi^{3}}\left(\frac{z_{0}(\xi)}{5}+\mu \xi\right), \quad a_{5}=\frac{4}{15 \xi^{3}} .
$$


При этом

$$
z_{0}=-5 \mu \xi+q \xi^{6 / 5}, \quad a_{6}=b_{6} \xi^{-18 / 5},
$$

где $q, b_{6}$ - произвольные постоянные.

Из уравнений $(18)$ и $(19)$ следует, что $a_{k}(\xi) \sim \xi^{3-(6 k / 5)}$. Тогда "общее" решение совместной системы $(18),(19)$ будет записываться в виде ряда

$$
v(z, \xi)=\frac{\xi^{3}}{\left(z-z_{0}\right)^{2}} \sum_{k=0}^{\infty} \frac{b_{k}\left(z-z_{0}\right)^{k}}{\xi^{6 k / 5}},
$$

где $b_{k}$-постоянные и $z_{0}=-5 \mu \xi+q \xi^{6 / 5}$, причем постоянные $q$ и $b_{6}$ являются произвольными.

4. Инвариантные решения уравнений иерархии Каупа. Рассмотрим иерархию систем уравнений $(2)$ при $n=2$. Первьй нетривиальный член этой иерархии система Каупа [4], [5]:

$$
\begin{aligned}
& u_{1 t_{2}}=\frac{1}{4} u_{2 x x x}+u_{1} u_{2 x}+\frac{1}{2} u_{2} u_{1 x} \\
& u_{2 t_{2}}=-u_{1 x}+\frac{3}{2} u_{2} u_{2 x} .
\end{aligned}
$$

Следуюшая система в этой иерархии определяется таким образом:

$$
\begin{aligned}
& u_{1 t_{3}}=-\frac{1}{4} u_{1 x x x}-\frac{3}{2} u_{1} u_{1 x}+\frac{3}{8} u_{2} u_{2 x x x}+\frac{9}{8} u_{2 x} u_{2 x x}+\frac{3}{2} u_{1} u_{2} u_{2 x}+\frac{3}{8} u_{2}^{2} u_{1 x} \\
& u_{2 t_{3}}=-\frac{1}{4} u_{2 x x x}+\frac{15}{8} u_{2}^{2} u_{2 x}-\frac{3}{2} u_{1} u_{2 x}-\frac{3}{2} u_{2} u_{1 x} .
\end{aligned}
$$

Построим совместное решение систем $(20),(21)$, инвариантное относительно преобразований (4), (11). В этом случае формулы преобразований (4), (11) вьглядят следуюшим образом:

$$
\begin{gathered}
\bar{u}_{1}=u_{1}-u_{2} \varepsilon+\varepsilon^{2}, \quad \bar{u}_{2}=u_{2}-2 \varepsilon, \quad \bar{x}=x+2 \varepsilon t_{2}+3 \varepsilon^{2} t_{3}, \\
\bar{t}_{2}=t_{2}+3 \varepsilon t_{3}, \quad \bar{t}_{3}=t_{3} .
\end{gathered}
$$

Дифференциальными инвариантами относительно образующей группы

$$
\mathbf{v}=-u_{2} \frac{\partial}{\partial u_{1}}-2 \frac{\partial}{\partial u_{2}}+3 t_{3} \frac{\partial}{\partial t_{2}}+2 t_{2} \frac{\partial}{\partial x}
$$

являются

$$
\xi=t_{3}, \quad z=x t_{3}-\frac{1}{3} t_{2}^{2}, \quad w_{1}=t_{3} u_{2}^{2}+\frac{4}{3} t_{2} u_{2}+\frac{4}{3} x, \quad w_{2}=u_{1}-\frac{1}{4} u_{2}^{2} .
$$

Стандартньми методами по аналогии с тем, как это делалось в предыдущем разделе, получаем подстановку

$$
u_{1}\left(x, t_{2}, t_{3}\right)=w_{2}(z, \xi)+\frac{1}{4 t_{3}^{2}}\left(v(z, \xi)-\frac{2 t_{2}}{3 t_{3}}\right)^{2}, \quad u_{2}\left(x, t_{2}, t_{3}\right)=\frac{-2 t_{2}+3 v(z, \xi)}{3 t_{3}}
$$

где $v=\left(\xi w_{1}-\frac{4}{3} z\right)^{1 / 2}$. Подстановка $(23)$ во второе уравнение системы Каупа дает соотношение

$$
w_{2 z}=\frac{v v_{z}}{\xi^{2}}+\frac{2}{3 \xi^{2}}
$$


Соотношение (24) можно проинтегрировать по переменной $z$. Выберем для удобства “постоянную интегрирования" $c_{1}(\xi)$ так, чтобы

$$
w_{2}=\frac{1}{\xi^{2}}\left(\frac{v^{2}}{2}+\frac{2 z}{3}+\frac{c_{1}(\xi)}{4}\right) .
$$

В свою очередь, подстановка (23) во второе уравнение системы (20) с учетом (25) дает уравнение с производной третьего порядка по $z$ на функцию $v=v(z, \xi)$. Интегрируя один раз по этой переменной, в результате получим

$$
v_{z z}=-\frac{1}{\xi^{4}}\left(2 v^{3}+\frac{8 z}{3} v+c_{1}(\xi) v\right)+c_{2}(\xi),
$$

где $c_{2}(\xi)$ - “постоянная интегрирования". Подстановка (23) во второе уравнение системы (21) с учетом (26) дает линейное эволюционное уравнение

$$
v_{\xi}=-\left(\frac{4 z}{3 \xi}+\frac{c_{1}(\xi)}{8 \xi}\right) v_{z}+\frac{2}{3 \xi} v .
$$

Наконец, подстановка (23) в первое уравнение системы (21) обращает это уравнение в тождество при условии, что $\xi c_{1}^{\prime}(\xi)=c_{1}(\xi)$. Нетрудно проверить, что условие совместности $v_{z z \xi}=v_{\xi z z}$ уравнений $(26)$ и $(27)$ выполняется, если $\xi c_{1}^{\prime}(\xi)=c_{1}(\xi)$ и $\xi c_{2}^{\prime}(\xi)=$ $-2 c_{2}(\xi)$. Решения этих уравнений запишем в виде

$$
c_{1}=8 \mu \xi, \quad c_{2}=\frac{\nu}{\xi^{2}},
$$

где $\mu, \nu$ - произвольные постоянные. Таким образом, доказано

УТВЕРЖДЕНИЕ 3. Подстановка

$$
\begin{aligned}
& u_{1}\left(x, t_{2}, t_{3}\right)=\frac{\left(9 v(z, \xi)-4 t_{2}\right) v(z, \xi)}{12 \xi^{2}}+\frac{6 z+18 \mu \xi+t_{2}^{2}}{9 \xi^{2}} \\
& u_{2}\left(x, t_{2}, t_{3}\right)=\frac{-2 t_{2}+3 v(z, \xi)}{3 \xi}, \quad \xi=t_{3}, \quad z=x t_{3}-\frac{1}{3} t_{2}^{2}
\end{aligned}
$$

дает совместное решение систем (20), (21), инвариантное относительно группы преобразований (22) при условии, что $v=v(z, \xi)$ удовлетворяет совместной системе уравнений

$$
\begin{gathered}
v_{z z}=-\frac{1}{\xi^{4}}\left(2 v^{3}+\frac{8}{3} z v+8 \mu \xi v\right)+\frac{\nu}{\xi^{2}} \\
v_{\xi}=-\left(\frac{4 z}{3 \xi}+\mu\right) v_{z}+\frac{2}{3 \xi} v
\end{gathered}
$$

где $\mu, \nu$ - произвольные постоянные.

Заметим, что при каждом фиксированном значении $\xi_{0} \neq 0$ уравнение $(29)$ является вторым уравнением Пенлеве на функцию $v(z)=v\left(z, \xi_{0}\right)$. Коэффициенты "общего" решения этого уравнения с простьм полюсом

$$
v(z)=\frac{1}{\left(z-z_{0}\right)} \sum_{k=0}^{\infty} a_{k}\left(z-z_{0}\right)^{k}
$$


определяются из рекурсионного соотношения вида

$$
(k+1)(k-4) a_{k}=f_{k}\left(a_{0}, \ldots, a_{k-1}\right), \quad k \geqslant 1,
$$

с резонансами при $k=-1$ и $k=4$, причем $a_{1}=0$. Подстановка (31) в уравнение $(29)$ дает

$$
a_{0}^{2}=-\xi^{4}, \quad a_{2}=\frac{4 a_{0}}{3 \xi^{4}}\left(\frac{z_{0}}{3}+\mu \xi\right), \quad a_{3}=\frac{2 a_{0}}{3 \xi^{4}}-\frac{\nu}{4 \xi^{2}}
$$

При этом

$$
z_{0}=-3 \mu \xi+q \xi^{4 / 3}, \quad a_{4}=b_{4} \xi^{-10 / 3},
$$

где $q, b_{6}$ - произвольные постоянные.

Из $(29)$ и $(30)$ следует, что $a_{k}(\xi) \sim \xi^{2-(4 k / 3)}$. "Общее” решение совместной системы (29), (30) тогда будет записьваться в виде ряда

$$
v(z, \xi)=\frac{\xi^{2}}{z-z_{0}} \sum_{k=0}^{\infty} \frac{b_{k}\left(z-z_{0}\right)^{k}}{\xi^{4 k / 3}}
$$

где $b_{k}$ - постоянные и $z_{0}=-3 \mu \xi+q \xi^{4 / 3}$, причем постоянные $q$ и $b_{4}$ являются произвольными.

Отметим, что оба линейных уравнения (19) и (30), описывающих деформацию решения соответствующего уравнения Пенлеве можно записать в единой форме, а именно

$$
v_{\xi}=A(z, \xi) v_{z}-\frac{1}{2} A_{z}(z, \xi) v
$$

где $A(z, \xi)=-(6 z /(5 \xi)+\mu)$ для первого уравнения Пенлеве и $A(z, \xi)=-(4 z /(3 \xi)+\mu)$ для второго уравнения Пенлеве.

5. Доказательство леммы. Обозначим

$$
p(\varepsilon)=p_{0}+\cdots+p_{n-1} \varepsilon^{n-1}+\varepsilon^{n}=\left.\left(\frac{1}{4} \partial+u_{1}+\frac{1}{2} u_{1 x} \partial_{x}^{-1}\right)\right|_{u_{1}=F_{1}(\overline{\mathbf{u}}, \varepsilon)} .
$$

В силу соотношений (3) можно записать

$$
\left.\Lambda(\varepsilon) \stackrel{\text { def }}{=} \Lambda[\mathbf{u}]\right|_{\mathbf{u}=\mathbf{F}(\overline{\mathbf{u}}, \varepsilon)}=\left(\begin{array}{ccccc}
0 & 0 & \ldots & 0 & p(\varepsilon) \\
-1 & 0 & \ldots & 0 & p^{\prime}(\varepsilon) \\
0 & -1 & \ddots & \vdots & \frac{1}{2 !} p^{\prime \prime}(\varepsilon) \\
\vdots & \ddots & \ddots & 0 & \vdots \\
0 & \ldots & 0 & -1 & \frac{1}{(n-1) !} p^{(n-1)}(\varepsilon)
\end{array}\right) .
$$

Так как матрица $A(\varepsilon)$ невырожденна, соотношение (5) можно переписать в виде

$$
A(\varepsilon)(\Lambda(0)+\varepsilon)=\Lambda(\varepsilon) A(\varepsilon)
$$

Коэффициенты $p_{i}$ являются операторами, однако очевидно, что при доказательстве выполнения соотношения (32) с этими коэффициентами можно оперировать как с числами. Для краткости биномиальные коэффищиенты обозначаем далее символом $C_{p}^{q}$. 
Имеем

$$
\begin{aligned}
& A(\varepsilon) \Lambda(0)=\left(\begin{array}{ccccc}
-\varepsilon & -\varepsilon^{2} & \ldots & -\varepsilon^{n-1} & p(\varepsilon)-\varepsilon^{n} \\
-1 & -C_{2}^{1} \varepsilon & \ldots & -C_{n-1}^{1} \varepsilon^{n-2} & p^{\prime}(\varepsilon)-C_{n}^{1} \varepsilon^{n-1} \\
0 & -1 & \ddots & -C_{n-1}^{2} \varepsilon^{n-3} & \frac{1}{2 !} p^{\prime \prime}(\varepsilon)-C_{n}^{2} \varepsilon^{n-2} \\
\vdots & \vdots & \ddots & \vdots & \vdots \\
0 & 0 & \ldots & -1 & \frac{1}{(n-1) !} p^{(n-1)}(\varepsilon)-C_{n}^{n-1} \varepsilon
\end{array}\right) \\
& \varepsilon A(\varepsilon)=\left(\begin{array}{cccccc}
\varepsilon & \varepsilon^{2} & \varepsilon^{3} & \ldots & \varepsilon^{n-1} & \varepsilon^{n} \\
0 & \varepsilon & C_{2}^{1} \varepsilon^{2} & \ldots & C_{n-2}^{1} \varepsilon^{n-2} & C_{n-1}^{1} \varepsilon^{n-1} \\
0 & 0 & \varepsilon & \ldots & C_{n-2}^{2} \varepsilon^{n-3} & C_{n-1}^{2} \varepsilon^{n-2} \\
\vdots & \vdots & \vdots & & \ddots & \vdots \\
0 & 0 & 0 & \ldots & 0 & \varepsilon
\end{array}\right) \\
& \Lambda(\varepsilon) A(\varepsilon)=\left(\begin{array}{cccccc}
0 & 0 & 0 & \ldots & 0 & p(\varepsilon) \\
-1 & -\varepsilon & -\varepsilon^{2} & \ldots & -\varepsilon^{n-2} & p^{\prime}(\varepsilon)-\varepsilon^{n-1} \\
0 & -1 & -C_{2}^{1} \varepsilon & \ldots & -C_{n-2}^{1} \varepsilon^{n-3} & \frac{1}{2 !} p^{\prime \prime}(\varepsilon)-C_{1}^{n-1} \varepsilon^{n-2} \\
\vdots & \vdots & \ddots & \vdots & \vdots & \vdots \\
0 & 0 & 0 & \ldots & -1 & \frac{1}{(n-1) !} p^{(n-1)}(\varepsilon)-C_{n-1}^{n-2} \varepsilon
\end{array}\right) .
\end{aligned}
$$

Сравнивая подиагонально элементы, нетрудно убедиться в выполнении соотношения (32). При этом нужно использовать соотношение

$$
C_{k}^{k-r}-C_{k-1}^{k-r-1}=C_{k-1}^{k-r}
$$

которое нетрудно проверить.

\section{СПИСОК ЦИТИРОВАННОЙ ЛИТЕРАТУРЫ}

[1] Овсянников Л. В. Групповой анализ дифференциальных уравнений. М.: Наука, 1978.

[2] Олвер П. Приложение групп Ли к дифференциальным уравнениям. М.: Мир, 1989.

[3] Alonso L. M. Schrödinger spectral problems with energy dependent potentials as sources of nonlinear Hamiltonian evolution equations // J. Math. Phys. 1980. V. 21. P. 2342-2349.

[4] Kaup D. J. Finding eigenvalue problems for solving nonlinear evolution equations // Progr. Theor. Phys. 1975. V. 54. P. 72-78.

[5] Matveev V. B., Yavor M. I. Solutions presque périodiques et à $N$-solutions de l'équation hydrodynamique nonlinéaire de Kaup // Ann. Inst. H. Poincaré. 1979. V. 31. P. 25-41.

Институт динамики систем и теории управления, г. Иркутск E-mail: svinin@icc.ru 